\title{
A Novel Thin-Film, Single-Junction Solar Cell Design' 1 to Achieve Power Conversion Efficiency above 30 Percent
}

\author{
Joseph Edward 0'Connor, Sherif Michael \\ Department of Electrical and Computer Engineering/Space Systems Academic Group, Naval Postgraduate School, Monterey, USA \\ Email: jeoconno1@nps.edu, michael@nps.edu
}

How to cite this paper: O'Connor, J.E. and Michael, S. (2016) A Novel Thin-Film, Single-Junction Solar Cell Design to Achieve Power Conversion Efficiency above 30 Percent. Materials Sciences and Applications, 7, 823-835.

http://dx.doi.org/10.4236/msa.2016.712063

Received: October 5, 2016

Accepted: December 10, 2016

Published: December 13, 2016

Copyright (c) 2016 by authors and Scientific Research Publishing Inc. This work is licensed under the Creative Commons Attribution International License (CC BY 4.0).

http://creativecommons.org/licenses/by/4.0/ (c) (i) Open Access

\begin{abstract}
The record efficiency for a thin-film, single-junction solar cell has remained static at $28.8 \%$ since 2012. This research presents a unique design that demonstrates potential to exceed record efficiency and approach the theoretical efficiency limit of $\sim 33.5 \%$. The findings of this study are significant, from an efficiency standpoint, and also because the cell design can be realized using existing fabrication methods that do not require complex, post-processing steps. In this study, a benchmark simulation is developed that closely resembles a high-efficiency, front-and-back contact cell. Intrinsic performance limiters are overcome by moving the emitter and front-contact to the back of the cell to eliminate electrical grid shading and improve optical performance. To further improve performance, the P-N junction formed by the emitter layer is removed from the model to allow selective Ohmic contacts to accept (reject) minority (majority) carriers as required. The design modifications improve open-circuit voltage, short-circuit current, and fill-factor which collectively boost efficiency above $30 \%$-primarily due to a $2 \%$ gain of incident irradiance and improved optical performance.
\end{abstract}

\section{Keywords}

Solar Cell, Back-Contacts, Gallium-Arsenide, Thin-Film

\section{Introduction}

The Shockley-Queisser (SQ) limit, proposed by W. Shockley and H. Queisser in 1961, is the upper theoretical efficiency of a single-junction solar cell (hereafter "cell") converter operating at $300 \mathrm{~K}$ under an incident spectrum approximated by a $6000 \mathrm{~K}$ blackbody

${ }^{1}$ Patents Pending. 
[1]. For a planar Gallium-Arsenide (GaAs) cell (band gap 1.42 electron-volts) the SQ limit is about $33.5 \%$. SQ limit research has expanded over the years to incorporate the AM1.5G spectrum, non-radiative recombination, and other variables; yet the limit has remained unchanged [2]. Given claims that manufacturing a $>30 \%$ efficient cell is possible, it seems improbable that record efficiency would stall at $\sim 25 \%$ from 1990-2007 and remain at $28.8 \%$ since 2012 [2]- [8]. In this paper, we present a novel design to exceed $30 \%$ efficiency by addressing intrinsic performance limitations of the high-efficiency (HE), thin-film, single-junction GaAs cell (hereafter "HE GaAs cell") presented in [3] [7] [8]. We begin by eliminating an estimated $2 \%$ front-grid shading loss [9] by employing all-back-contact (hereafter "back-contact") technology that has achieved success with silicon cells [10] [11]. Then we remove the P-N junction formed by the emitter layer to enhance carrier mobility and allow a selective Ohmic contact to accept (reject) minority (majority) carriers as required [6]. These design improvements boost model efficiency above $30 \%$ and suggest that record efficiency for HE GaAs cells can be improved.

\section{Modeling Semiconductor Devices in Silvaco ${ }^{\circledR}$ Atlas $^{\mathrm{TM}}$}

Silvaco is an industry leader for modeling semiconductor devices, and Atlas is the preferred simulator for predicting electrical characteristics of cell structures under bias conditions. Atlas constructs two-dimensional (2D) or 3D structures with internal grids that generate intersections called nodes. Continuity equations, current-density $J_{S C}$ equations, and Poisson's equation are solved at each node to achieve convergence and simulate the transport of charge carriers in a cell. Device modeling provides insight into the physical phenomena of a cell and displays data in a visual platform [12].

Since it is not cost-effective to design cells by trial and error, modeling can be useful to investigate ideas before investing time and money to build a prototype. The down-side of modeling is the uncertainty involved in accounting for all physical processes that occur in a cell. Simplifications, numerical methods, and other factors guarantee that a model will never exactly simulate the physical behavior of a cell. Nevertheless, we show that a high-level of confidence can be attained for a given model by carefully accounting for key design parameters, benchmarking model behavior to experimental results, and making single-variable adjustments to predict the behaviors of a new design.

\subsection{Modeling a High-Efficiency, Thin-Film, Single-Junction, Gallium-Arsenide Solar Cell with Front and Back Contacts}

We begin by assembling design characteristics from [2]-[8] to develop a model that closely resembles the performance of the HE GaAs cell from [3] [8]. We make no assertion that the structure shown in Figure 1 is an exact representation of the cell; however, careful consideration was given to matching design variables with experimental data as we show in this section. It is the opinion of the authors that the cell structure shown in Figure 1 is the product of an industry that focuses on the optimization of front-contact 


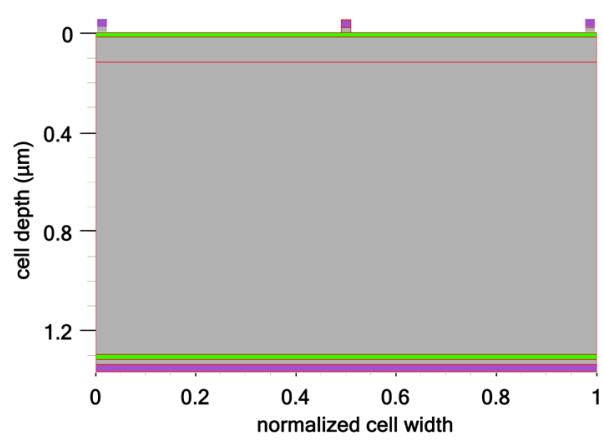

(a)

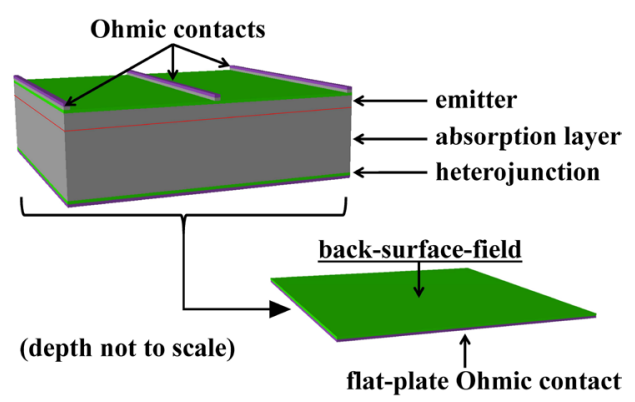

(b)

Figure 1. (a) 2D model representation of the front-contact HE GaAs cell presented in [3] [8]; (b) $3 \mathrm{D}$ model representation of the HE GaAs cell presented in [3] [8].

grid dimensions to balance trade-offs associated with shading and series resistance. We note that most researchers utilize front-contact technology to achieve record efficiency; however, we believe the benefits derived from back-contact technology can outweigh the drawbacks as with the current record for large-area crystalline silicon cells and Sun Power ${ }^{\oplus}$ Corporation's industry-leading commercial cell [3]. Both back-contact designs make the case for exploring the technology as a suitable structure for thin-film GaAs cells.

The HE GaAs cell's foundation is high-quality GaAs manufactured via a metal-organic chemical vapor deposition (MOCVD) and an epitaxial-lift-off (ELO) process. Since the cell is only $\sim 1 \mu \mathrm{m}$ thick and material quality is excellent, efficient electron transport and minimal bulk recombination is achieved. Additionally, passivation of the cell's front and back surfaces yields very low surface-recombination velocity which, among other factors, contributes to the highest open-circuit voltage $V_{O C}$ recorded for a single-junction cell. The back-contact is $>90 \%$ reflective, which enhances photon recycling through radiative recombination and band-edge absorption. A broadband anti-reflection coating (ARC) encompasses the spectral response of the cell $(0.3-0.9 \mu \mathrm{m})$ with some room for improvement at shorter wavelengths as noted in [8]. A front-surface electrical grid with estimated $2 \%$ coverage is derived from [8], which corresponds with the coverage of a similar HE cell reported in [9]. Grid shading loss is combined with $4.5 \%$ ARC reflectivity/window layer absorption losses to yield a total $J_{S C}$ reduction of $\sim 6.5 \%$, which corresponds with [5]. We assume a minority carrier lifetime of $\sim 50$ nanoseconds for an absorption layer doped to $\sim 10^{17} \mathrm{~cm}^{-3}$, which is compounded by photon recycling [i.e., the process whereby photons are emitted through radiative recombination and subsequently produce another electron-hole-pair (EHP)] to produce "effective" lifetimes of 10x or greater [8] and corresponds to values reported in [13] [14] [15].

Auger recombination is the process whereby an electron-hole-pair (EHP) transfers energy and momentum to a third carrier via non-radiative recombination [16]. Auger recombination is modeled as

$$
R_{\text {auger }}=C_{n}\left(p n^{2}-n n_{i}^{2}\right)+C_{p}\left(n p^{2}-p n_{i}^{2}\right)
$$


where $C_{n}\left(C_{p}\right)$ is the auger coefficient for electrons (holes), and $n_{i}$ is the intrinsic electron concentration. Coefficient values ranging from $7 \times 10^{-30} \mathrm{~cm}^{6} \cdot \mathrm{s}^{-1}$ to $1.6 \times 10^{-29}$ $\mathrm{cm}^{6} \cdot \mathrm{s}^{-1}$ are reported in literature which often distinguishes between direct and indirect auger recombination [16]. This work combines direct and indirect auger recombination effects and sets $C_{n}$ and $C_{p}$ to $7 \times 10^{-30} \mathrm{~cm}^{6} \cdot \mathrm{s}^{-1}$ to match HE GaAs cell performance parameters. Reference [5] uses the same value and reports auger recombination as $\sim 6 \%$ of total recombination in a $\mathrm{HE} \mathrm{GaAs}$ cell simulation, which suggests that the mechanism should not be ignored.

Radiative or optical recombination is the process whereby an electron from the conduction band combines with a hole in the valence band to release a photon. This type of recombination is dominant in direct band gap semiconductors (e.g., GaAs) and is essentially the inverse of optical generation. Radiative recombination is modeled as

$$
R_{\text {rad }}=B\left(n p-n_{i}^{2}\right)=B n_{i}^{2}\left[\exp \left(\frac{E_{F_{n}}-E_{F_{p}}}{k T}\right)-1\right] \approx B n_{i}^{2} \exp \left(\frac{E_{F_{n}}-E_{F_{p}}}{k T}\right)
$$

where $B$ is the intrinsic radiative recombination coefficient, $E_{F_{n}}-E_{F_{p}}$ is the energy difference between EHP quasi-Fermi levels, $k$ is Boltzmann's constant, and $T$ is the operating temperature. Radiative recombination contributes to photon recycling in the HE GaAs cell, which determines the effective minority carrier lifetime. Experimental measurements of $B$ range from $\sim 10^{-11} \mathrm{~cm}^{3} \cdot \mathrm{s}^{-1}$ to $\sim 10^{-10} \mathrm{~cm}^{3} \cdot \mathrm{s}^{-1}$ in literature [15] [16] [17] [18] [19], and we adopt $10^{-11} \mathrm{~cm}^{3} \cdot \mathrm{s}^{-1}$ to match $\mathrm{HE} \mathrm{GaAs} \mathrm{cell} \mathrm{performance.}$

Shockley-Read-Hall (SRH) or "trap-assisted" recombination is a two-step process whereby an electron (hole) occupies an energy level in the band gap and then recombines with a hole (electron). The energy traps may be intentional (i.e. due to doping) or unintentional (i.e., due to defects). If SRH recombination occurs at a surface or interface due to intermediate energy levels caused by dangling bonds or lattice mismatch, it is often referred to as "surface" or "interface" recombination. SRH recombination is modeled as

$$
R_{S R H}=\frac{n p-n_{i}^{2}}{\tau_{p}\left[n+n_{i} \exp \left(\frac{E_{\text {trap }}}{k T}\right)\right]+\tau_{n}\left[p+n_{i} \exp \left(\frac{-E_{\text {trap }}}{k T}\right)\right]}
$$

where $\tau_{n}\left(\tau_{p}\right)$ is the electron (hole) minority carrier lifetime, and $E_{\text {trap }}$ is the energy difference between the impurity "trap" located in the band gap and the intrinsic Fermi levels. In this work, we assume a single trap level which corresponds to the most efficiency recombination center. Distributed trap states may be modeled; however, separate SRH statistics are required.

Carrier mobility is dependent upon doping concentration. We set electron and hole mobility for intrinsic GaAs (at $300 \mathrm{~K}$ ) to 8500 and $400 \mathrm{~cm}^{2} \cdot \mathrm{V}^{-1} \cdot \mathrm{s}^{-1}$, respectively [17], and employ a concentration-mobility (CONMOB) dependent model to account for reduced mobility due to doping. Fermi statistics are implemented to account for reduced carrier concentrations in the heavily doped emitter and cap layers adjacent to Ohmic contacts; however, we note that model output did not change significantly when using 
Boltzmann statistics. Surface recombination velocities are modeled at $45 \mathrm{~cm}-\mathrm{s}^{-1}$ and 10 $\mathrm{cm}^{-1} \mathrm{~s}^{-1}$ for external surfaces and internal interfaces, respectively, which corresponds with velocities given in [5] [17] [20].

Experimentally derived refractive index values (real and imaginary) are critical for accurate model performance, and extinction coefficients near the band edge are especially important since they determine the extent that photons are absorbed and "recycled" after radiative recombination events-a primary design consideration for $\mathrm{HE}$ cell performance. We found that a widely referenced optical database [18] contained few reference points at the band edge; thus, we compiled information from [21] [22] [23] [24] to cross-check measurements, identify outliers, and assemble a dense measurement sequence. The resulting collection of refractive indices instilled confidence that photon absorption and reflection were accurately accounted for in the model.

The authors of [5] and [17] modeled lumped resistance to match HE GaAs cell fill-factors; however, we opted to model resistivity since the parameter characterizes resistance independently of contact area and improves model accuracy. Ohmic contacts are often used to achieve very low resistivity required for HE operation, typically $<10^{-3}$ $\Omega-\mathrm{cm}^{2}$ for the back-contact (full-coverage) and $<10^{-5} \Omega-\mathrm{cm}^{2}$ for the front-contact (partial coverage). References [25] and [26] assert that semiconductor doping on the order of $10^{19} \mathrm{~cm}^{-3}$ enables Ohmic contact where tunneling is the dominant transport mechanism. Reference [26] surveys experimental data from $n$-type GaAs/metal contacts and $p$-type GaAs/metal contacts to conclude that $10^{19} \mathrm{~cm}^{-3}$ doping for $p$-type GaAs is typical, whereas $4 \times 10^{18} \mathrm{~cm}^{-3}$ is more realistic for $n$-type GaAs. However, [27] reports $n$-type GaAs doped to $10^{19} \mathrm{~cm}^{-3}$ on $\mathrm{Au} / \mathrm{Pt} / \mathrm{Ti}$ to achieve $1.1 \times 10^{-6} \Omega-\mathrm{cm}^{2}$ resistivity, and [9] reports $n$-type GaAs doped to $6.5 \times 10^{18} \mathrm{~cm}^{-3}$ on $\mathrm{Pd} / \mathrm{Ge} / \mathrm{Au}$ to achieve $3.6 \times 10^{-6}$ $\Omega-\mathrm{cm}^{2}$ resistivity. We adopt conservative doping concentrations of $5 \times 10^{18} \mathrm{~cm}^{-3}$ for the $n$-type, front-contact cap layer with a corresponding resistivity of $\sim 10^{-5} \Omega-\mathrm{cm}^{2}$, and $10^{19}$ $\mathrm{cm}^{-3}$ for the $p$-type, back-contact cap layer with a corresponding resistivity of $\sim 10^{-3}$ $\Omega-\mathrm{cm}^{2}$. Incidentally, the low contact resistivities of the cell described in [9] produced an excellent fill-factor FF which contributed to record efficiency in 2009. We note the technological challenges and high cost associated with manufacturing a front-contact with low-resistivity and point out that the problem is avoided entirely when using backcontact technology.

Various works cited in this paper employ models to modify key design parameters in order to determine the impact on cell performance. Authors compare model output parameters with experimental measurements $\left(J_{s c}, V_{o c}, F F\right.$, and efficiency $\left.\eta\right)$ to instill confidence in their simulations; however, model output is not compared with experimentally measured external quantum efficiency (EQE). We assert that matching $V_{O C}$, $J_{S C,} F F$, and $\eta$ of a physical cell is not sufficient and that models should also reproduce the EQE curve to ensure that key variables such as window layer absorption and ARC performance are accounted for in the model. To this end, we compare cell performance parameters and EQE traces from [8] and [18] to our model output as shown in Figure 2 (a) and Figure 2(b), respectively. 


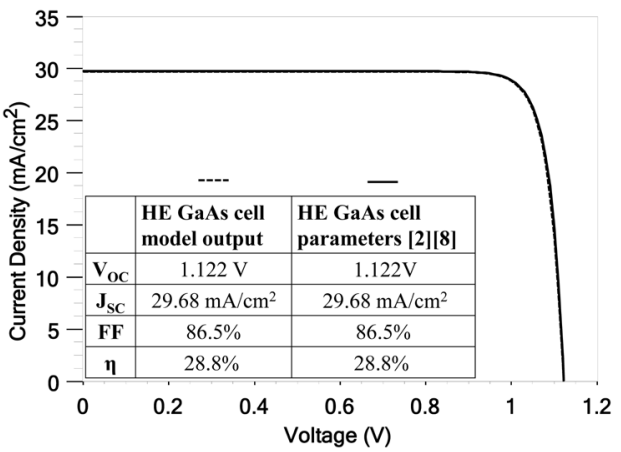

(a)

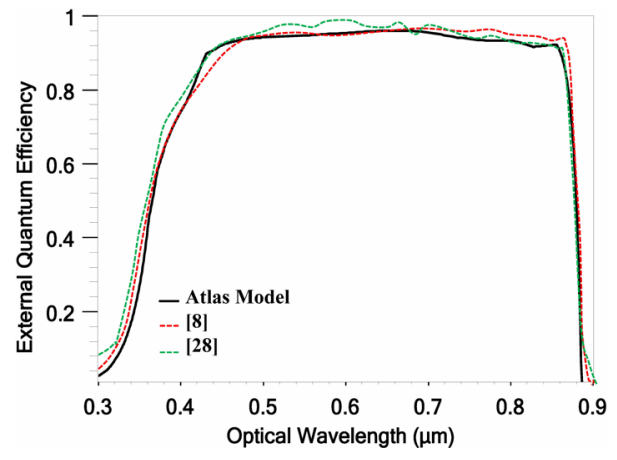

(b)

Figure 2. (a) Comparison of HE GaAs cell model output parameters and $J$ - $V$ curve to measurements presented in [8] [28]; (b) Comparison of HE GaAs cell model EQE curve to measurements presented in [8] [28]. Referenced EQE curves were traced and scaled to fit the plot.

\subsection{Modeling a High-Efficiency, Thin-Film, Single-Junction Gallium-Arsenide Solar Cell with Back-Contacts}

Figure 2 establishes confidence that the model sufficiently resembles HE GaAs cell performance as presented in [3] [8]. To maintain the integrity of the model as we experiment with a new design, we alter a single variable and move the emitter (and associated front-contact) to the back of the device. To this end, we reduce the coverage of the back-contact and back-surface-field (BSF) to $40 \%$ to make room for the emittercontact which occupies the remaining $60 \%$. All other variables are held constant except for emitter-contact resistivity which is increased from $10^{-5} \Omega-\mathrm{cm}^{2}$ to $10^{-3} \Omega-\mathrm{cm}^{2}$ since ultra-low front-grid resistivity is not required in a back-contact design. $2 \mathrm{D}$ and $3 \mathrm{D}$ structures of the GaAs, Back-surface Alternating-Contact (GaAs-BAC) cell design are shown in Figure 3(a) and Figure 3(b), respectively. Note that the BSF is split to occupy each side of the back-surface in order to establish symmetry and enable 2D simulation, which decreases model run-time.

The GaAs-BAC cell model exceeded the HE GaAs Cell model for performance categories shown in Figure 4(a). $V_{O C}$ improved 1.0\%, $J_{S C}$ improved 1.4\%, FF improved $2.7 \%$, and $\eta$ improved 5.2\%. If we categorize the GaAs-BAC cell structure as a $\sim 1 \mu \mathrm{m}$ thick, planar, untextured cell with good back-surface reflectivity ( 90\%), [2] indicates that $F F$ and $V_{O C}$ are close to their theoretical limits of $89.1 \%$ and $1.15 \mathrm{~V}$, respectively. Conversely, $J_{S C}$ has room to improve to its theoretical limit of $31.6 \mathrm{~mA} / \mathrm{cm}^{2}$. We focus on improving $J_{S C}$ and identifying the possible causes of $\sim 1.5 \mathrm{~mA} / \mathrm{cm}^{2}$ loss by setting front-surface reflectivity to $0 \%$ and changing refractive index values in the window layer to make it completely transparent. The adjustments improve $J_{S C}$ to $31.53 \mathrm{~mA} / \mathrm{cm}^{2}$, which suggests that ARC reflection and window layer absorption are the main contributors to current-density loss. An examination of the EQE curve in Figure 4(b) further

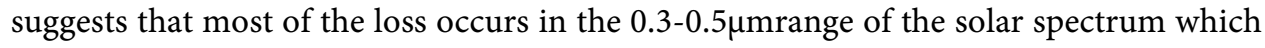
is almost entirely absorbed near the front-surface of the cell. We test the hypothesis by evaluating optical generation $G$ from the front-to-back surface of the cell and spectral generation $g$ at various depths as shown in Figure 5(a) and Figure 5(b). Analysis of the 


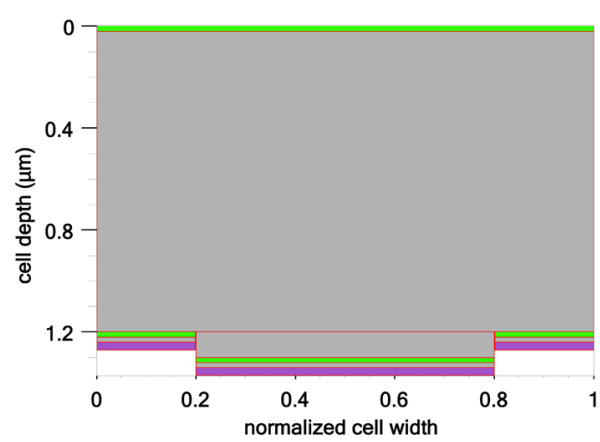

(a)

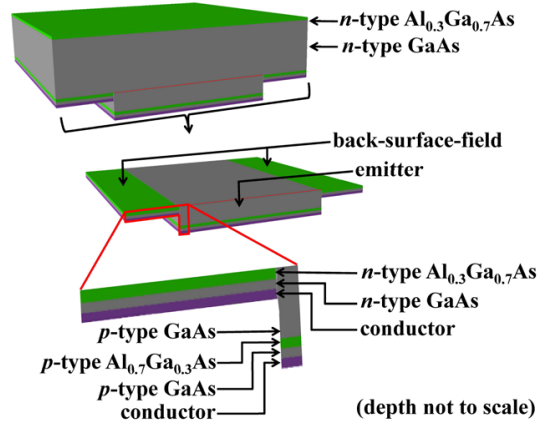

(b)

Figure 3. (a) GaAs-BAC cell model 2D structure. Note that the BSF occupies both sides of the structure to achieve symmetry and enable accurate 2D modeling; (b) GaAs-BAC cell model 3D structure with key parts labeled. Front-surface and back-surface heterojunctions reduce surface recombination. A "thin" insulating layer occupies space between the emitter and BSF contacts to prevent electrical shorting. Both $2 \mathrm{D}$ and $3 \mathrm{D}$ modeling were used in this work, and results were very similar; therefore, $2 \mathrm{D}$ modeling was adopted to decrease simulation time.

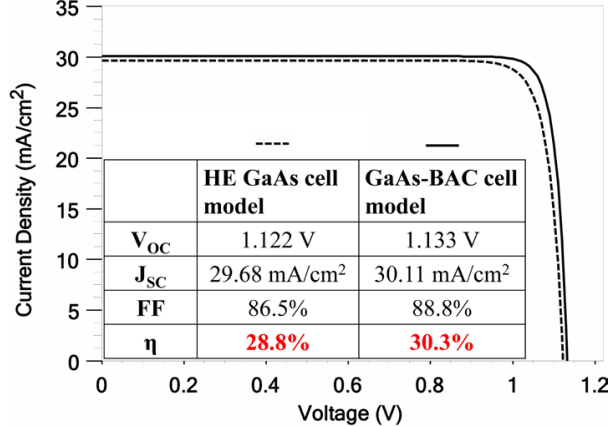

(a)

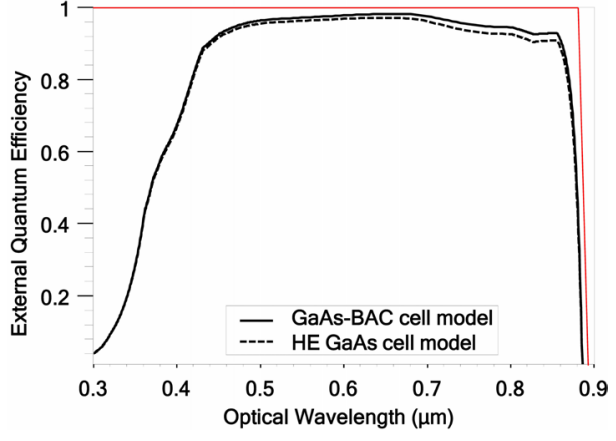

(b)

Figure 4. (a) J-V curves and output parameters of the HE GaAs cell model vs. the GaAs-BAC cell model; (b) EQE curves of the HE GaAs cell model vs. the GaAs-BAC cell model. Ideal EQE response shown in red. The space (gap) between black and red lines indicates loss due to recombination. Higher loss is noted in the $0.3-0.5 \mu \mathrm{m}$ range. Lower loss is noted above $0.5 \mu \mathrm{m}$.

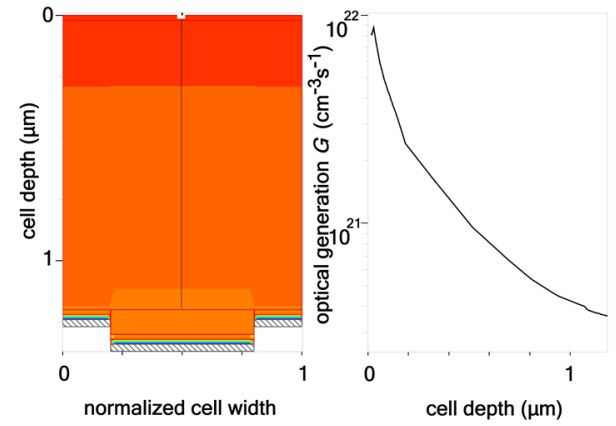

(a)

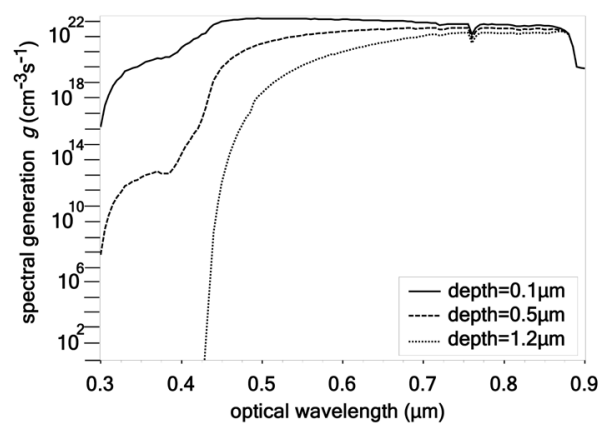

(b)

Figure 5. (a) GaAs-BAC cell model optical generation measured from the front-to-back surface of the cell; ${ }^{2}$ (b) GaAs-BAC cell model spectral generation measured at various depths $d$ within the cell.

${ }^{2}$ Reference Appendix for more information on Figure 5. 
plots confirm that $0.3-0.5 \mu \mathrm{m}$ wavelengths are indeed absorbed in the front $\sim 10 \%$ of the cell; hence, $J_{S C}$ may be improved by reducing reflection and improving window layer transparency in that spectral range. Further examination of Figure 4(b) reveals less severe losses above $0.5 \mu \mathrm{m}$ that can be attributed to external luminescence loss and non-radiative recombination loss during carrier transport [8]. We estimate external luminescence loss at $<2 \%$, which is indicative of a "good" HE cell [4]; however, we note that carrier transport could be enhanced by improving carrier mobility-a topic addressed in the next section.

\subsection{Modeling an "Emitter-Less" High-Efficiency, Thin-Film, Single-Junction, Gallium-Arsenide Solar Cell with Back-Contacts}

Examination of the GaAs-BAC cell model structure shown in Figure 3 identified two regions of high doping concentration and low carrier mobility-the emitter layer and contact-cap layers. Contact-cap layers are constrained to high doping concentration to achieve Ohmic contact with metal; however, the emitter layer is thicker than contact layers and has a greater impact on carrier mobility. At $300 \mathrm{~K}$, electron and hole mobility in the GaAs-BAC cell model is reduced to $\sim 3500 \mathrm{~cm}^{2} \cdot \mathrm{V}^{-1} \cdot \mathrm{s}^{-1}$ and $\sim 350 \mathrm{~cm}^{2} \cdot \mathrm{V}^{-1} \cdot \mathrm{s}^{-1}$, respectively, for an emitter-layer doping concentration of $\sim 10^{17} \mathrm{~cm}^{-3}$ [29]. The band diagrams in Figure 6(a) and Figure 6(b) represent an " $n$-on- $p$ " GaAs-BAC cell design (i.e. an $n$-type absorption layer interfaced with a $p$-type emitter layer). ${ }^{3}$ The built-in voltage at cell depth $d=1.2 \mu \mathrm{m}$ shown in Figure 6(a) is designed to separate EHPs; however, the electrostatic potential also reduces $V_{O C}$ Reference [6] asserts that the built-in voltage (P-N junction) is not required for certain cell designs. In the case of the GaAs-BAC cell, minority carrier lifetime is relatively high compared to the distance

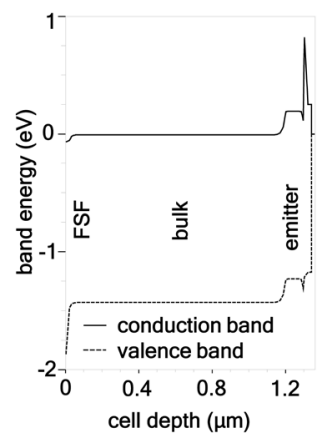

(a)

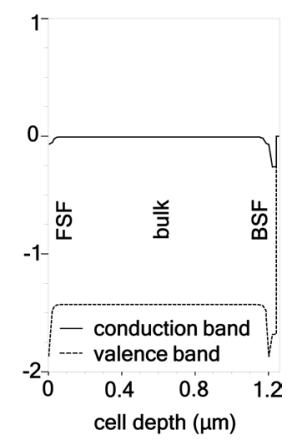

(b)

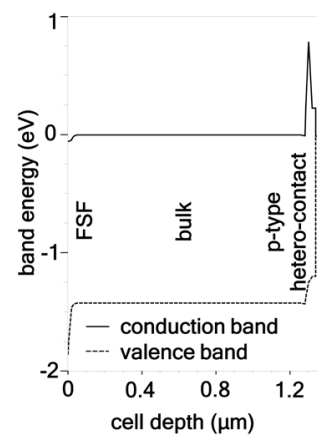

(c)

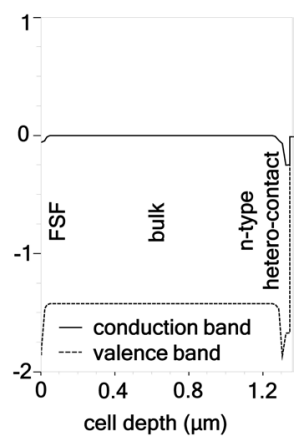

(d)

Figure 6. (a) GaAs-BAC cell model band diagram traced from the front-surface through the emitter. The characteristic P-N junction is shown at depth $d=1.2 \mu \mathrm{m}$; (b) GaAs-BAC cell model band diagram traced from the front-surface through the BSF; (c) Emitter-less GaAs-BAC cell model band diagram traced from the front-surface through the $p$-type hetero-contact that accepts minority carriers (holes) and rejects majority carriers (electrons); note the absence of a P-N junction; (d) Emitter-less GaAs-BAC cell model band diagram traced from the front-surface through the $n$-type hetero-contact that accepts majority carriers (electrons) and rejects minority carriers (holes).

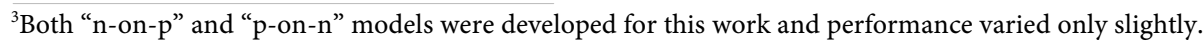


that carriers must diffuse to reach the back-contact; hence, the cell may not require a P-N junction to keep EHPs separated. Furthermore, the p-type hetero-contact at the back-surface is specifically designed to accept (reject) minority (majority) carriers, which implies that the purpose of the emitter may be redundant, or even unnecessary. Therefore, we remove the emitter from the GaAs-BAC cell model and note the results on the band diagrams shown in Figure 6(c) and Figure 6(d).

The "emitter-less" GaAs-BAC cell allows minority carriers (holes) to diffuse freely in the cell with a high probability of capture at the $p$-type hetero-contact (for an " $n$-on- $p$ " cell). Note that the diffusion gradient required to drive carriers to a contact is very small ( $\sim 1$ millivolt) and reduces $V_{O C}$ only slightly. In fact, the model shows that $V_{O C}$ (measured to the nearest millivolt) is not reduced, whereas $J_{S C}$ and $F F$ improve slightly as shown in Figure 7(a). Another benefit of removing the emitter is the elimination of the GaAs-BAC cell's structural "offset" as shown in Figure 7(b). This is an important attribute because a "flat" back-surface is easier to manufacture via the MOCVD/ELO process.

\section{Conclusion and Future Work}

This research demonstrates the advantage of modeling a novel solar cell design to investigate performance impacts prior to investing time and money to build a prototype. We developed a benchmark model of a thin-film, GaAs solar cell from [3] [8] and changed the model structure to investigate how the cell would perform with back-surface contacts. The design alternation significantly improved $V_{O C}, J_{S C}, F F$, and $\eta$-mainly due to a $2 \%$ incident irradiance gain from the removal of the front-contact. Analysis of the GaAs-BAC cell simulation identified an area of reduced carrier mobility in the heavily-doped emitter region, which was subsequently removed to analyze whether a "selective" hetero-contact would maintain or even improve performance. We found that $J_{S G}, F F$, and $\eta$ improved slightly, which supports the hypothesis proposed in [6] that certain cell designs do not require a $\mathrm{P}-\mathrm{N}$ junction if hetero-contacts are implemented. We conclude that the relatively long lifetime and comparatively short diffusion length of minority carriers in the GaAs-BAC cell enable efficient operation without a

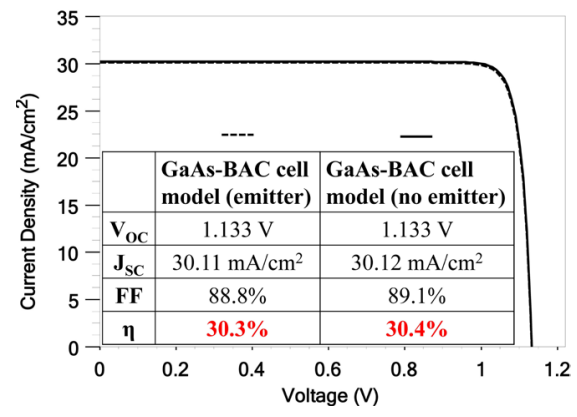

(a)

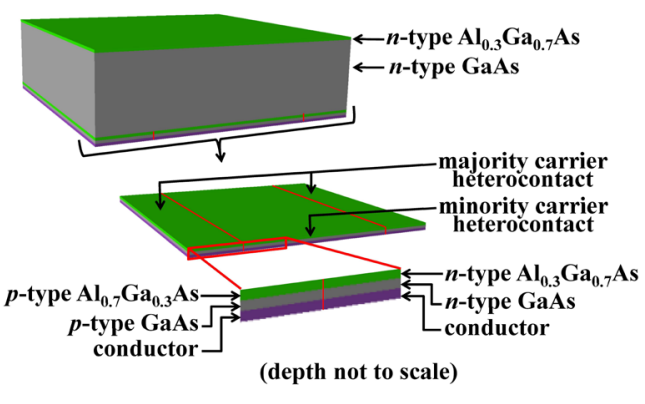

(b)

Figure 7. (a) J-V curves and output parameters of the GaAs-BAC cell model vs. the emitter-less GaAs-BAC cell model; (b) 2D structure of the emitter-less GaAs-BAC cell model. 
P-N junction.

Future research will concentrate on design parameter optimization to further improve cell efficiency. Specifically, the benefits of random texturing on the front-andback surfaces to promote internal reflection and photon recycling will be investigated. Additionally, high-temperature operation and "radiation-hardness" will be examined to evaluate the cell's potential for employment aboard spacecraft.

Patents are pending for designs presented in this paper [30] [31].

\section{References}

[1] Shockley, W. and Queisser, H. (1961) Detailed Balance Limit of Efficiency of P-N Junction Solar Cells. Journal of Applied Physics, 32, 510-520. https:/doi.org/10.1063/1.1736034

[2] Miller, O., Yablonovich, E. and Kurtz, S. (2012) Strong Internal and External Luminescence as Solar Cells Approach the Shockley-Queisser Limit. IEEE Journal of Photovoltaics, 2, 303311. https:/doi.org/10.1109/JPHOTOV.2012.2198434

[3] Green, M., Emery, K., Hishikawa, Y., Warta, W. and Dunlop, E. (2016) Solar Cell Efficiency Tables (Version 47). Progress in Photovoltaics Research and Applications, 24, 3-11. https:/doi.org/10.1002/pip.2728

[4] Miller, O. and Yablonovich, E. (2013) Photon Extraction, the Key Physics for Approaching Solar Cell Efficiency Limits. Proceedings of SPIE Conference Optics + Photonics, San Diego, 25-29 August 2013, 880807-1-10. https:/doi.org/10.1117/12.2024592

[5] Wang, X., Khan, M., Gray, J., Alam, M. and Lundstrom, M. (2016) Design of Gaas Cells Operating Close to the Shockley-Queisser Limit. IEEE Journal of Photovoltaics, 3, 737-744. https:/doi.org/10.1109/JPHOTOV.2013.2241594

[6] Yablonovich, E. (2011) The Optoelectronic Physics That Just Broke the Efficiency Record in Solar Cells. http://energyseminar.stanford.edu

[7] Kayes, B., et al. (2011) 27.6\% Conversion Efficiency-A New Record for Single Junction Solar Cells under 1 Sun Illumination. Proceedings of IEEE Photovoltaic Specialist Conference, Seattle, 19-24 June 2011, 4-8. https:/doi.org/10.1109/pvsc.2011.6185831

[8] Kayes, B., et al. (2012) Light Management in Single-Junction III-V Solar Cells. SPIE Optics + Photonics Conference Plenary Presentation, San Diego, 12-16 August 2012.

[9] Bauhuis, G., Mulder, P., Haverkamp, E., Huijben, J. and Schermer, J. (2009) 26.1\% ThinFilm Gaas Cell Using Epitaxial Lift-Off. Solar Energy Materials \& Solar Cells, 93, 14881491. https:/doi.org/10.1016/j.solmat.2009.03.027

[10] O’Connor, J. and Michael, S. (2016) Impact of Operating Temperature and AbsorptionLayer Thickness on All-Back-Contact Solar Cell Efficiency. Proceedings of EU Photovoltaic Solar Energy Conference, Munich, 20-24 June 2016, 983-986.

[11] O'Connor, J. and Michael, S. (2016) Design and Optimization of a Novel Back-Contact Solar Cell in Silvaco ${ }^{\circledR}$ Space Photovoltaic Research and Technology Conference, Cleveland, 20-22 September 2016, 1-3.

[12] SILVACO ${ }^{\circledR}$ (2016) ATLAS $^{\mathrm{Tm}}$ User's Manual. https://dynamic.silvaco.com/dynamicweb/jsp/downloads/DownloadManualsAction.do?req =silentmanuals\&nm=atlas

[13] Lumb, M., Steiner, M., Geisz, J. and Walters, R. (2014) Incorporating Photon Diffusion Recycling into the Analytical Drift-Diffusion Model of High Efficiency Solar Cells. Journal of Applied Physics, 116, 116-125. https:/doi.org/10.1063/1.4902320

[14] Walker, A., et al. (2015) Impact of Photon Recycling on GaAs Solar Cell Designs. IEEE 
Journal of Photovoltaics, 5, 1636-1645. https:/doi.org/10.1109/JPHOTOV.2015.2479463

[15] Steiner, M., et al. (2013) Effects of Internal Luminescence and Internal Optics of Voc and Jsc of III-V Solar Cells. IEEE Journal of Photovoltaics, 3, 1437-1442. https:/doi.org/10.1109/JPHOTOV.2013.2278666

[16] Steiauf, D., Kioupakis, E. and van de Walle, C., (1993) Auger Recombination in GaAs from First Principles. ACS Photonics, 1, 643-646. https:/doi.org/10.1021/ph500119q

[17] IoffePhysico-Technical Institute (2016) Characteristics and Properties of Semiconductor Materials. http://www.ioffe.ru/SVA/NSM/

[18] Lush, L., et al. (1991) Determination of Minority Carrier Lifetimes in N-Type GaAs and their Implications for Solar Cells. Proceedings of IEEE Photovoltaic Specialist Conference, Las Vegas, 7-11 October 1991, 182-187. https:/doi.org/10.1109/pvsc.1991.169205

[19] Ahrenkiel, R. (1992) Measurement of Minority-Carrier Lifetime by Time-Resolved Photoluminescence. Solid State Electronics, 35, 239-250. https:/doi.org/10.1016/0038-1101(92)90228-5

[20] Sopra, S.A. (2016) GaAs Optical Data. http://www.sspectra.com/sopra

[21] Skauli, T., et al. (2003) Improved Dispersion Relations for GaAs and Applications to Nonlinear Optics. Journal of Applied Physics, 94, 6447-6455. https:/doi.org/10.1063/1.1621740

[22] Jellison Jr., G. (1992) Optical Functions of GaAs, GaP, and Ge Determined by Two-Channel Polarization Modulation Ellipsometry. Optical Materials, 1, 151-160. https:/doi.org/10.1016/0925-3467(92)90022-F

[23] Aspnes, D., Kelso, S., Logan, R. and Bhat, R. (1986) Optical Properties of AlGaAs. Journal of Applied Physics, 60, 754-767. https:/doi.org/10.1063/1.337426

[24] Kachare, A., Spitzer, W. and Fredrickson, J. (1976) Refractive Index of Ion-Implanted GaAs. Journal of Applied Physics, 47, 4209-4212. https:/doi.org/10.1063/1.323292

[25] Schroder, D. and Meier, D. (1984) Solar Cell Contact Resistance-A Review. IEEE Transactions on Electronic Devices, 31, 637-647. https:/doi.org/10.1109/T-ED.1984.21583

[26] Baca, A., Ren, F., Zolper, J., Briggs, R. and Pearton, S. (1997) A Survey of Ohmic Contacts to III-V Compound Semiconductors. Thin Solid Films, 308, 599-606. https:/doi.org/10.1016/S0040-6090(97)00439-2

[27] Ren, R., Cho, A., Sivco, D., Pearton, S. and Abernathy, C. (1994) Use of Sn-Doped GaAs for Non-Alloyed Ohmic Contacts to HEMTs. Electronic Letters, 30, 912-914. https:/doi.org/10.1049/el:19940599

[28] Green, M., Emery, K., Hishikawa, Y., Warta, W. and Dunlop, E. (2012) Solar Cell Efficiency Tables (Version 40). Progress in Photovoltaics: Research and Applications, 20, 606-614. https:/doi.org/10.1002/pip.2267

[29] Blakemore, J. (1982) Semiconducting and Other Major Properties of Gallium Arsenide. Journal of Applied Physics, 53, 123-181. https:/doi.org/10.1063/1.331665

[30] Michael, S. and O’Connor, J. (2016) GaAs Solar Cell with Back-Surface, Alternating-Contacts. US Patent Application 15/207,128.

[31] O’Connor, J. and Michael, S. (2016) Emitter-Less, Back-Surface, Alternating-Contact Solar Cell. US Patent Application 15/282,460. 


\section{Appendix}

We confirmed that the solar cell simulation was working correctly by calculating spectral generation at an arbitrary wavelength and optical generation (for all wavelengths in the spectral response) at particular depths using experimentally-derived extinction coefficients. The calculations were then compared to model output to verify performance as shown below.

The absorption coefficient $\alpha$ as a function of energy for direct band gap materials is defined as

$$
\alpha(h v)=A\left(h v-E_{G}\right)^{\frac{1}{2}}
$$

where $A$ is a material-dependent constant, $h$ is Planck's constant, $v$ is the frequency, and $E_{G}$ is the material band gap. For solar cell analysis, it is convenient to define $\alpha$ as a function of wavelength $\lambda$ as in

$$
\alpha(\lambda)=\frac{4 \pi k}{\lambda}
$$

where $k$ is the extinction coefficient and the imaginary portion of the index of refraction. Equation (5) is calculated at an arbitrary wavelength $\lambda=600 \mathrm{~nm}$ (where $k=0.214$ ) to give $4.5 \times 10^{6} \mathrm{~m}^{-1}$.

Photon flux $\phi$ at the cell's front-surface is defined as

$$
\phi(\lambda)=P(\lambda) \frac{\lambda}{h c}
$$

where $P$ is the spectral power density and $c$ is the speed of light. Integrating the AM1.5G solar spectrum yields $P=1000 \mathrm{~W} \cdot \mathrm{m}^{-2}$; hence, (5) gives $\phi=3 \times 10^{21} \mathrm{~m}^{-2} \cdot \mathrm{s}^{-1}$ at the front-surface of the cell.

The spectral generation rate $g$ is defined as

$$
g(\lambda, d)=[1-R(\lambda)] \eta \phi(\lambda) \alpha(\lambda) \exp [-\alpha(\lambda) d]
$$

where $R$ is the front-surface reflectivity, $\eta$ is the internal quantum efficiency, and $d$ is the depth (of interest) in the cell. Equation (7) calculated at $\lambda=600 \mathrm{~nm}$ and $d=1.2 \mu \mathrm{m}$ gives $7 \times 10^{19} \mathrm{~cm}^{-3} \cdot \mathrm{s}^{-1}$. Now the optical generation rate $G$ may be defined as

$$
G(d)=\int_{\lambda_{1}}^{\lambda_{2}} g(\lambda, d) \mathrm{d} \lambda
$$

which gives $3.6 \times 10^{20} \mathrm{~cm}^{-3} \cdot \mathrm{s}^{-1}$ at $d=1.2 \mu \mathrm{m}$, measured over the spectral response of the cell $\left(\lambda_{1}=300 \mathrm{~nm}\right.$ and $\left.\lambda_{2}=900 \mathrm{~nm}\right)$. Comparing calculations to the model output shown in Figure A1, we conclude that the simulation accurately predicts carrier generation. 


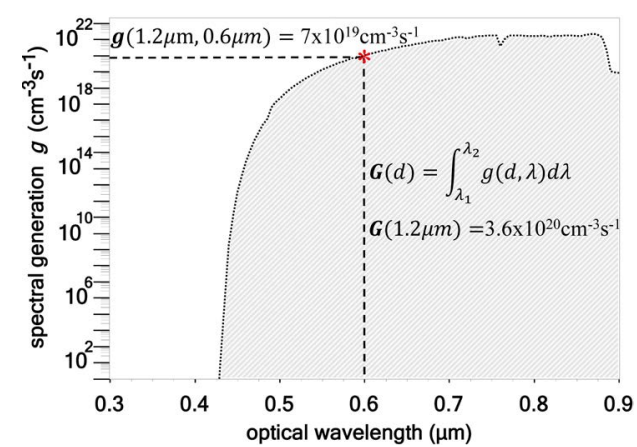

(a)

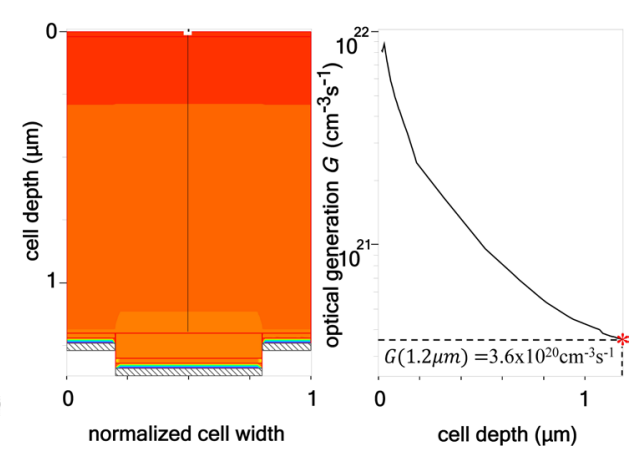

(b)

Figure A1. (a) GaAs-BAC cell model spectral generation at cell depth $d=1.2 \mu \mathrm{m}$; the red asterisk plots spectral generation $g$ at $\lambda=0.6 \mu \mathrm{m}(600 \mathrm{~nm})$; the spectral generation curve is integrated to yield optical generation $G$ over the spectral response range (300 nm - $900 \mathrm{~nm}$ ); (b) GaAs-BAC cell model optical generation $G$ plotted from the front-surface to the back-surface of the cell. The red asterisk plots optical generation $G$ at cell depth $d=1.2 \mu \mathrm{m}$. Spectral and optical generation values plotted in the figures correspond with hand calculations and confirm that the model accurately predicts carrier generation.

\section{Submit or recommend next manuscript to SCIRP and we will provide best service for you:}

Accepting pre-submission inquiries through Email, Facebook, LinkedIn, Twitter, etc.

A wide selection of journals (inclusive of 9 subjects, more than 200 journals)

Providing 24-hour high-quality service

User-friendly online submission system

Fair and swift peer-review system

Efficient typesetting and proofreading procedure

Display of the result of downloads and visits, as well as the number of cited articles

Maximum dissemination of your research work

Submit your manuscript at: http://papersubmission.scirp.org/

Or contact msa@scirp.org 